\title{
Good practice guidelines for biomarker discovery from array data: a case study for breast cancer prognosis
}

\author{
Jie Cheng ${ }^{1 *}$, Joel Greshock ${ }^{2}$, Leming Shi ${ }^{3}$, Shu Zheng ${ }^{4}$, Alan Menius ${ }^{1}$, Kwan Lee ${ }^{1}$ \\ From IEEE International Conference on Bioinformatics and Biomedicine 2012 \\ Philadelphia, PA, USA. 4-7 October 2012
}

\begin{abstract}
Background: Biomarker discovery holds the promise for advancing personalized medicine as the biomarkers can help match patients to optimal treatment to improve patient outcomes. However, serious concerns have been raised because very few molecular biomarkers or signatures discovered from high dimensional array data can be successfully validated and applied to clinical use. We propose good practice guidelines as well as a novel tool for biomarker discovery and use breast cancer prognosis as a case study to illustrate the proposed approach.

Results: We applied the proposed approach to a publicly available breast cancer prognosis dataset and identified small numbers of predictive markers for patient subpopulations stratified by clinical variables. Results from an independent cross-platform validation set show that our model compares favorably to other gene signature and clinical variable based prognostic tools. About half of the discovered candidate markers can individually achieve very good performance, which further demonstrate the high quality of feature selection. These candidate markers perform extremely well for young patient with estrogen receptor-positive, lymph node-negative early stage breast cancers, suggesting a distinct subset of these patients identified by these markers is actually at high risk of recurrence and may benefit from more aggressive treatment than cur-rent practice.
\end{abstract}

Conclusion: The results show that by following good practice guidelines, we can identify highly predictive genes in high dimensional breast cancer array data. These predictive genes have been successfully validated using an independent cross-platform dataset.

\section{Introduction}

The goal of biomarker discovery from high dimensional array data is to find an individual or a set of genes (or any other molecular variables) whose expression pattern can predict certain phenotype or clinical outcome. Biomarker discovery holds the promise for advancing personalized medicine as the biomarkers can help match patients to optimal treatment and thus improve patient outcomes. During the past 15 years, numerous biomarkers and gene signatures have been published in the literature. However, few of these biomarkers can be successfully validated and applied in clinical setting, which have caused serious concerns in biomedical research community [1].

${ }^{1}$ Quantitative Sciences, GlaxoSmithKline, Collegeville, PA 19426, USA

Full list of author information is available at the end of the article
The lack of success in biomarker discovery is mainly due to three issues.

(1) Many published gene signatures cannot be validated independently. This is mainly due to flawed data analysis. For example, fail to keep completely untouched validation data or improperly use cross-validation technique can all cause overly optimistic results being reported.

(2) Some gene signatures do not have additional clinical benefit over known clinical variables even though the gene signatures can be validated. For example, gene signatures discovered from a breast cancer patient population including both ER + and ER- patients for predicting treatment response could probably end up approximating ER status, an important clinical variable for predicting treatment response. 
(3) Some gene signatures contain large numbers of genes making them difficult to be applied in clinical setting. These gene signatures may include lot of unimportant genes due to the inefficiency of the standard feature selection techniques. For example, standard t test will either miss or rank certain important features below many non predictive features, if these important features do not conform to normality.

The first two issues have been addressed in [1,2]. To address the third issue, we developed a two-way filtering based method to parsimoniously identify the most informative features from different types of distributions. Our method selects features by searching for the desired thresholds of a pair of statistics that are used to filter features. For any pair of thresholds, the features that satisfy both thresholds are used to build a diagonal linear discriminate analysis $(D L D A)$ classifier [3]. When choosing the pair of statistics, we choose one that is more efficient at detecting strong signals such as mean difference test, and a second that is more efficient at controlling signal to noise ratio such as classical t-test or Mann-Whitney $U$ test. By varying the thresholds of these two statistics in certain steps within their acceptable ranges, we can achieve various tradeoffs and control the size of the feature sets. The method and other supporting functions are implemented in a Java based tool called Array Data Analyzer (ADA). The detailed method is described in [4].

Compared to standard feature selection tools, our $A D A$ tool has the following advantages.

(1) Because our approach does not select features based on any single statistic or fixed tradeoff of two statistics, it can adapt to different data and parsimoniously identify informative features from different distributions. Experimental result presented in [4] shows that our approach yields much smaller models on average when compared to other standard approaches, yet achieving similar or better performance.

(2) By using grid-search to explore different tradeoffs of a pair of statistics, our tool is very flexible to address different feature selection requirements in real world situations. For example, researchers at times are willing to sacrifice performance in order to gain other logistic properties, such as smaller numbers of features and larger fold change. These properties enable more tractable assays for clinical use (e.g. qPCR). By visually examining the performance at different combinations of cut points of the two statistics and checking the size of feature sets, researchers can decide which feature set would be best to use.

(3) Our Java based tool is very efficient to run even though it conducts rigorous cross validation and grid search to find optimal feature sets. For example, finding an optimal gene signature from microarray gene expression dataset with 130 samples takes about 50 seconds on a modest laptop PC (Dell Latitude E4300 with 2GB of RAM). (Searching through 200 combinations of the threshold pairs of the two statistics; using 5 times 5 fold cross validation (CV) to measure performance of each combination).

The $A D A$ tool has been applied to various GSK drug discovery projects [5]. It has also been applied to the FDA MAQC-II project [6] by the GSK data analysis team (DAT). The GSK DAT achieved highest mean area under the receiver operating characteristic curve (AUR$O C C)$ across all 11 endpoints among the participating DATs. Using $A D A$ tool, we also achieved the second place in the "The Sage Bionetworks/DREAM breast cancer prognosis challenge 2012" (https://sagebionetworks. jira.com/wiki/display/BCC/Home) by simply identifying 8 genes from the provided training set.

As a case study, we performed detailed prognostic analyses on the van de Vijver breast cancer dataset [7]. An independent cross-platform validation set TRANSBIG [8] was then used to validate our findings. The results show that our 20-gene signature as well as many of the discovery individual prognostic biomarkers can achieve comparable or better performance compared to the clinical or gene signature based prognostic scores. These discovered biomarkers have the potential to be used in clinical settings to identify a subset of the lymph-nodenegative (Node-) and estrogen-receptor-positive (ER+) patients who are at a higher risk of relapse.

\section{Methods}

\section{The $A D A$ tool}

The $A D A$ tool allows users to choose different pairs of statistics to perform the two-way filtering. In this paper, the default pair of non parametric statistics is used, which are the mean difference test and the Mann-Whitney $\mathrm{U}$ test. More detailed information of the ADA tool is given in [4].

\section{Data preprocessing}

van de Vijver dataset and TRANSBIG dataset were downloaded from the public domain. For all datasets generated from Affymetrix platforms, if a gene intensity value is smaller than 40 , we floor it to 40 . The log transformed ex-pression values are used for analysis.

\section{Scoring and classification of validation data sets using 20 gene signature}

For the discovered genes that can be successfully mapped from Agilent Hu25K platform to Affymetrix HG-U133A, we arbitrarily decided to keep top 10 genes from each direction (i.e., over expression $\rightarrow$ poor prognosis and 
over expression $\rightarrow$ good prognosis) to validate. If a gene can be mapped to multiple probe sets of the Affymetrix HG-U133A chip, we use the probe set that has the largest interquartile range (IQR). To avoid bias, the scoring of validation samples using the 20 gene signature was done without any scaling or tuning. We simply calculated the scores by summing up the log expression values of genes of one direction and subtracting those of genes of the other direction. To classify the samples into "good prognosis" and "poor prognosis", we use zero as the threshold.

\section{Results}

As a case study, we use the van de Vijver data set [7] to discover prognostic biomarkers for various patient subsets stratified by clinical variables. The markers discovered from the lymph node negative patient cohort are subsequently evaluated using an independent cross-platform dataset: TRANSBIG [8]. Table 1 summarizes the training and independent validation data sets. Both data sets are publicly available.

Model training and prognostic biomarker discovery using van de Vijver dataset

The van de Vijver data set contains samples from 295 patients with stage I and II breast cancer. The gene expression data was generated using Agilent $\mathrm{Hu} 25 \mathrm{~K}$ platform. A subset of the samples was used to develop a 70-gene signature for predicting breast cancer early relapse [9]. To make the data less noisy, we only included patients who developed metastases in $<5$ years and those who remained disease free for $>10$ years (i.e. we removed the patients who developed metastases between 5-10 years) in the biomarker discovery phase. This left 78 patients who developed metastases within 5 years (poor prognosis) and 68 patients who remain disease free for at least 10 years. Besides analyzing all the data together, we also utilized the clinical information (ER status and lymph node status) to create subgroups of samples in order to search for prognostic markers within these clinical subgroups.

By using the $A D A$ tool, we were able to generate gene lists of various sizes for different subgroups of patients. After studying the gene lists, we found that many of the predictive markers were indeed more sig-nificant in certain subgroups. For example, the ER+/lymph node+ group harbors unique genes that are predictive of relapse. The prognosis of this group of patients was also more predictable (based on nested CV results) compared to that of the other groups. Some of the discovered genes are listed in Table 2.

Table 1 Summary of the two data sets involved in this experiment

\begin{tabular}{lcc}
\hline Data set & van de Vijver & TRANSBIG \\
\hline Purpose in this experiment & biomarker discovery & biomarker validation \\
Microarray platform & Agilent Hu25K & Affymetrix HG-U133A \\
No. of Patients & 295 & 198 \\
Age & $<53$ & $<61 ;$ mean $=46$ \\
ER status (pos/neg) & $226 / 69$ & $134 / 64$ \\
Lymph node (pos/neg) & $144 / 151$ & $0 / 198$ \\
Systemic treatment & some & none \\
Data location or GEO accession & http://bioinformatics.nki.nl/data.php & GSE7390 \\
\hline
\end{tabular}

Table 2 Candidate markers identified from the van de Vijver data set using the proposed method

\begin{tabular}{|c|c|c|c|c|}
\hline Group & $\begin{array}{l}\text { Sample size } n \text { (good } \\
\text { prog + poor prog) }\end{array}$ & $\begin{array}{l}\text { Nested CV } \\
\text { AUROCCperformance }\end{array}$ & $\begin{array}{l}\text { Feature list (high expression } \rightarrow \text { poor } \\
\text { prognosis) }\end{array}$ & $\begin{array}{l}\text { Feature list (high expression } \rightarrow \\
\text { good prognosis) }\end{array}$ \\
\hline $\begin{array}{l}\text { All } \\
\text { patient }\end{array}$ & $146(68+78)$ & $0.73(0.04)$ & $\begin{array}{l}\text { BIRC5, CCNB2, CENPA, TK1, CCNE2, } \\
\text { DKFZP762E1312, PRC1, STK15, SLC16A3, BUB1 }\end{array}$ & $\begin{array}{l}\text { CEGP1, SLC11A3, C4A, ZNF145, } \\
\text { MATN3, PGR, RAI2, DLX2 }\end{array}$ \\
\hline ER+ & $107(57+50)$ & $0.76(0.05)$ & H1F2, COX6C, H2BFB, CCNE2, BLVRB & $\begin{array}{l}\text { FST, DIO3, NTN4, DLX2, MATN3, } \\
\text { COL3A1 }\end{array}$ \\
\hline Node+ & $64(30+34)$ & $0.80(0.06)$ & $\begin{array}{l}\text { H1F2, H2BFB, HA2FO, H2AFA, HABFB, } \\
\text { KFZp762E1312, H2BFS }\end{array}$ & $\begin{array}{l}\text { LTF, NTN4, HML2, PER1, DMBT1, } \\
\text { ODZ2, WNT5A, SEMA3C }\end{array}$ \\
\hline Node- & $82(38+44)$ & $0.72(0.06)$ & PRAME, FADSD6, TK1, TSSC3, CTSL2, BUB1 & $\begin{array}{l}\text { CEGP1, ESR1, CYP4B1, SEC14L2, } \\
\text { TBX3-iso, ZNF145 }\end{array}$ \\
\hline $\begin{array}{l}\text { ER } \\
+/ \text { Node } \\
+\end{array}$ & $50(26+24)$ & $0.83(0.06)$ & $\begin{array}{l}\text { H1F2, H2BFB, H2AFP, H2AFA, H2BFB, COX6C, } \\
\text { MSMB, BLVRB, , BCAS1 }\end{array}$ & $\begin{array}{l}\text { LTF, LAMB3, C4A, NTN4, PTPRK, } \\
\text { RTN1 }\end{array}$ \\
\hline
\end{tabular}




\section{Independent cross platform validation using TRANSBIG dataset}

To validate the candidate biomarkers discovered from van de Vijver data set, we download the TRANSBIG data set (198 patients with mean age equals 46), which is based on the Affymetrix HG-U133A platform instead of the Agilent $\mathrm{Hu} 25 \mathrm{~K}$ platform. An original objective of TRANSBIG data set was to validate the 70-gene signature and it is similar to the van de Vijver data set in term of patient age distribution - i.e., both data sets contains young breast cancer patients. Because all patients of the TRANSBIG data set are lymph node negative (Node-) patients, we evaluate the candi-date biomarkers discovered from the Node- patients.

We picked the top 20 genes from our Node- gene list that can be mapped from Agilent $\mathrm{Hu} 25 \mathrm{~K}$ to Af-fymetrix HG-U133A. The performance of the 20 gene-signature as well as each of the individual gene was measured using AUROCC based on commonly used endpoints: time to distant metastasis(TDM) at 5 years and 10 years. Because $70 \%$ of Node- patients in the training data belong to the subgroup Node-/ER+, in addition to measure the performance on the whole validation sets (consisting of Node- patients), we also measured the performance on the Node-/ER+ subset of patients. Both results are shown in Table 3 , which also includes the performance of two commonly used clinical scores (Nottingham Prognostic Index Score [10] and Adjuvant! 10 year overall survival score [11]), three clinical variables (tumor grade, tumor size and age), two known markers (ESR1 and MKI67) and three gene signatures (16-gene signature [12], 70-gene signature [9] and 76-gene signature [13]).

From the result we can see that our 20-gene signature performs better than other gene signature or clinical variable based prognostic factors. Most of the 20 candidate genes can also be successfully validated. About half of the 20 genes can individually achieve similar or better performance compared to clinical variable based risk scores, which further shows that the proposed approach can parsimoniously select high quality features.

To further illustrate the added value of these markers over the traditional clinical criteria, we use survival curves to compare the 20-gene signature to the Adjvant! 10 year overall survival probability, which is based on known clinical markers. Figure 1 plots four Kaplan-Meier curves by dividing the TRANSBIG patients into four risk groups based on the predictions from 20-gene signature and the Adjvant! tool. The plots show that our 20-gene signature is superior in predicting cancer outcome, especially for the 66 patients (33\% of the 198 samples) shown in the dotted blue survival curve where the 20-gene signature predicts good outcome and Adjvant! predicts poor outcome. This curve indeed traces the curve in
Table 3 Validation performance (AUROCC) of the candidate biomarkers in TRANSBIG data sets

\begin{tabular}{|c|c|c|c|c|}
\hline \multirow[t]{3}{*}{ Prognostic factors } & \multicolumn{4}{|c|}{ TRANSBIG } \\
\hline & \multicolumn{2}{|c|}{ TDM at $5 y$ rs } & \multicolumn{2}{|c|}{ TDM at $10 \mathrm{yrs}$} \\
\hline & Node- & $\begin{array}{c}\text { Node-/ER } \\
+\end{array}$ & Node- & $\begin{array}{c}\text { Node-/ER } \\
+ \\
\end{array}$ \\
\hline 202705_at(CCNB2) & 0.74 & 0.83 & 0.72 & 0.80 \\
\hline 209642_at(BUB1) & 0.71 & 0.81 & 0.70 & 0.78 \\
\hline 204962_s_at(CENPA) & 0.69 & 0.84 & 0.69 & 0.79 \\
\hline 203362_s_at(MAD2L1) & 0.68 & 0.75 & 0.67 & 0.71 \\
\hline 202095_s_at(BIRC5) & 0.67 & 0.78 & 0.65 & 0.74 \\
\hline 210074_at(CTSL2) & 0.65 & 0.64 & 0.65 & 0.64 \\
\hline 209803_s_at(PHLDA2, TSSC3) & 0.61 & 0.62 & 0.59 & 0.61 \\
\hline 202338_at (TK1) & 0.61 & 0.69 & 0.60 & 0.64 \\
\hline 204086_at(PRAME) & 0.61 & 0.62 & 0.57 & 0.58 \\
\hline 202218_s_at (FADSD6) & 0.50 & 0.49 & 0.50 & 0.45 \\
\hline 210096_at(CYP4B1) & 0.71 & 0.78 & 0.70 & 0.74 \\
\hline 205883_at(ZNF145) & 0.69 & 0.75 & 0.66 & 0.71 \\
\hline 219197_s_at(SCUBE2, CEGP1) & 0.66 & 0.66 & 0.63 & 0.59 \\
\hline 214053_at(ERBB4) & 0.66 & 0.72 & 0.67 & 0.74 \\
\hline 208305_at(PGR) & 0.65 & 0.66 & 0.64 & 0.66 \\
\hline 219682_s_at(TBX3) & 0.63 & 0.66 & 0.63 & 0.65 \\
\hline 204541_at(SEC14L2) & 0.63 & 0.65 & 0.62 & 0.59 \\
\hline 206091_at(MATN3) & 0.61 & 0.55 & 0.61 & 0.56 \\
\hline 202554_s_at(GSTM3) & 0.60 & 0.62 & 0.58 & 0.59 \\
\hline 219440_at(RAl2) & 0.59 & 0.59 & 0.56 & 0.56 \\
\hline Our 20-gene signature & 0.73 & 0.83 & 0.70 & 0.79 \\
\hline 16-gene signature & 0.71 & 0.79 & 0.69 & 0.73 \\
\hline 70-gene signature & 0.68 & NA & NA & NA \\
\hline $\begin{array}{l}\text { Nottingham Prognostic Index } \\
\text { Score }\end{array}$ & 0.67 & 0.68 & 0.66 & 0.66 \\
\hline $\begin{array}{l}\text { Adjuvant! Online } 10 \text { year OS } \\
\text { prob. }\end{array}$ & 0.66 & 0.64 & 0.67 & 0.63 \\
\hline 76-gene signature & 0.65 & 0.68 & 0.62 & 0.64 \\
\hline Tumor grade & 0.64 & 0.63 & 0.62 & 0.62 \\
\hline Tumor Size & 0.63 & 0.65 & 0.63 & 0.64 \\
\hline 212021_s_at(MKI67) & 0.62 & 0.70 & 0.65 & 0.70 \\
\hline 205225_at (ESR1) & 0.58 & 0.59 & 0.57 & 0.61 \\
\hline Age & 0.53 & 0.47 & 0.52 & 0.51 \\
\hline
\end{tabular}

There are two AUROCC numbers for each gene at each endpoint. The first number is from the whole validation set with $100 \%$ Node- patients; the second is from the Node-/ER+ subset of the validation set. The numbers in bold font are significant at $95 \%$ confidence level. The top portion of the table contains 10 genes of one direction (over expression $\rightarrow$ poor prognosis). The middle portion contains 10 genes of the opposite direction (over expression $\rightarrow$ good prognosis). The bottom portion contains our signature based on all 20 genes and other prognostic factors. The performance of the 70-gene signature for the TRANSBIG data set is copied from [8]. The performance of the 76-gene signature is based on binary prediction of "good prognosis" and "poor prognosis" for each patient. Among the listed 20 genes, three genes (CENPA, GSTM3 and CEGP1) were included in the 70-gene signatures [9] and four genes (BIRC5, PGR, SCUBE2 and CTSL2) were included in the 16-gene signature. There is no overlap between our 20-gene signature and the 76-gene signa-ture.

solid blue where the patients were predicted to have good outcome by both classifiers.

It is also worth noting that the 20-gene signature and the top performing genes based on the whole vali-dation 


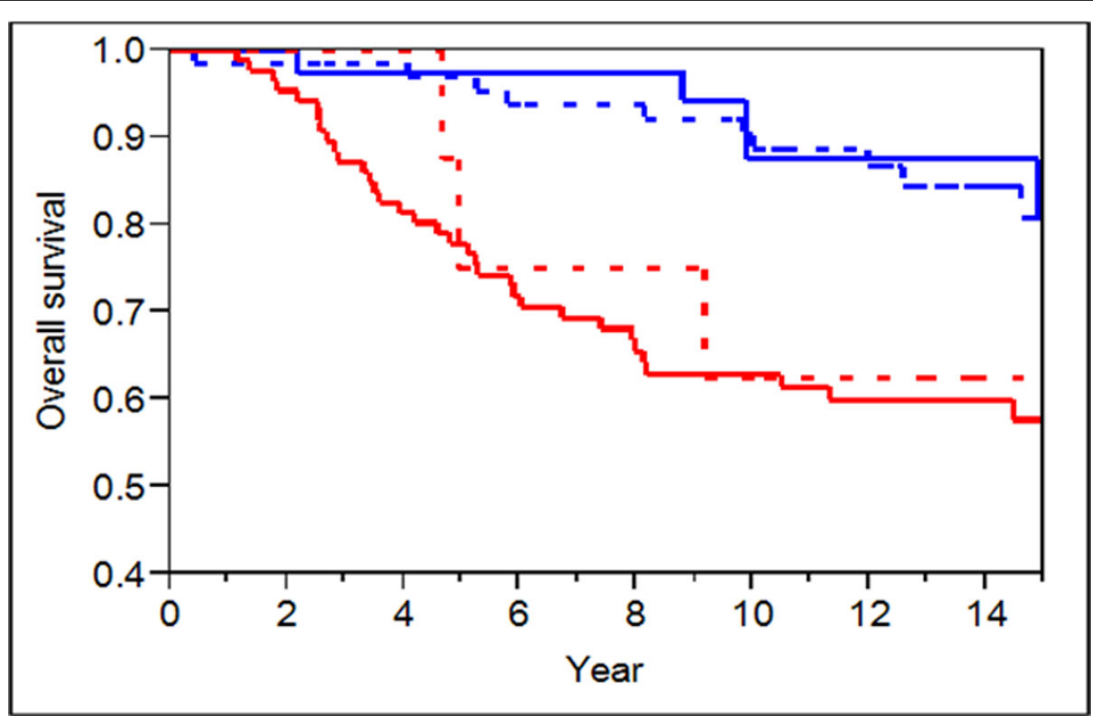

Figure 1 Using Kaplan-Meier curves to compare 20-gene signature with the Adjvant! 10 year overall sur-vival score. Solid blue curve is for the risk group that both 20-gene signature and Adjvant! predict good prognosis $(n=38)$; Dotted blue curve is for the risk group that 20-gene signature predicts good prognosis and Adjvant! predicts poor prognosis( $n=66)$; Dotted red curve is for the risk group that 20-gene signature predicts poor prognosis and Adjvant! predicts good prognosis $(n=8)$; Solid red curve is for the risk group that both classifiers predict poor prognosis $(n=86)$.

set have even better performance when validated using Node-/ER+ patients only. For example, for end point TDM at 5 years, the 20 -gene signature can achieve $A U R$ OCC 0.83; and the genes CCNB2, BUB1, CENPA, BIRC5 and CYP4B1 can each achieve AUROCC above 0.78 .

\section{Discussion}

Using the breast cancer prognosis experiment, the $A D A$ tool was able to effectively discover both biologically relevant and class predictive genes. The tool has two important features: (a) because the model performance estimation is based on rigorous nested cross-validation, the tool does not return any feature when the dataset does not produce a strong signal. (b) It has a unique iterative procedure that attempts to find all important genes, which can be used for further analyses, such as pathway and ontology analysis.

When doing biomarker discovery using the van de Vijver datasets, our approach relies heavily on robust cross validation to control over fitting rather than leaving out an artificial validation set. This is a more efficient way to use the relatively small number of samples - artificially defining a small validation set is unlikely to achieve much due to the large test data variability and reduced power of model development in the smaller training set. However, it is crucial that the cross-validation is done properly. For example, the feature selection must be performed within each run of cross validation; and the nested cross validation is often required when evaluating model performance (i.e., outer loop CV for model performance evaluation and inner loop CV for model parameter tuning). As we can see here, the proper cross-validation procedures can often be quite computationally expensive. This is one of the reasons that a simple modeling technique such as $D L D A$ is preferred. We believe the best way to gain better performance is through improving performance of feature selection, rather than tuning modeling parameters of complex models. Complicated learning schemes can make proper cross-validation too computational expensive to run. Without proper crossvalidation, the result can be overly optimistic. Important guidelines for biomarker discovery are presented in Richard Simon's work [2].

As shown in Table 2, when sample size allows, we try to discover biomarkers for patient subpopulations stratified by clinical variables. This also assures us that the discovered biomarkers indeed have added value over the traditional clinical variables, rather than approximating the clinical variables.

To validate our findings from the van de Vijver data, we used the cross-platform validation dataset TRANSBIG. In addition to evaluate our 20-gene signature, we also evaluate individual genes. By doing so, we can carry forward those validated genes for further clinical validations. Table 3 shows that most of the 20 genes can be validated using the TRANSBIG data set and some of the discovered candidate genes can individually achieve similar or better performance compared to the state-of-the-art breast cancer prognostic tests, which suggest that our 20-gene signature contains mostly highly predictive genes. It is very likely 
that large gene signatures contain many unimportant genes. We believe that a promising way to improve prediction accuracy is to combine gene signatures with traditional clinical variables. This can be achieved by developing gene signatures containing a small number of genes for each clinically homogeneous subset of patients.

Table 3 also shows that for Node-/ER+ patients of younger age (as those in TRANSBIG dataset), there are a number of genes that can predict cancer recurrence at high accuracy. This suggests that there is a distinct subgroup of Node-/ER+ patients who have high risk of relapse and should be treated more aggressively. Our 20-gene signature is acquired from analyzing one public dataset for one patient subpopulation (Node-). We plan to analyze other datasets to refine this predictive gene list and develop gene lists for other patient subpopulations.

Based on our past experience, we are confident that biomarker discovery can play important role in ad-vancing personalized medicine if the study design and data analysis is done properly. We would like to propose the following good practice guidelines for biomarker discovery. Most of the points have already been made in $[1,2]$.

(1) Make sure no information leak from validation set. If a separate validation set is available, one need to make sure that the validation set is not used in any way in feature filtering or model building. The same rule also applies to cross validation.

(2) Model performance evaluation and model parameter tuning cannot be done using the same cross va-lidation loop. In such case, nested cross validation is needed (i.e., outer loop CV for model performance evaluation and inner loop CV for model parameter tuning).

(3) When sample size is not very large, cross validation is a preferred validation technique.

(4) Consider patient stratification using known clinical variables.

(5) Use simple modeling techniques. Simple models performs well [3] and are easy to train and easy to un-derstand.

(6) When choosing feature filtering or feature selection methods, do not automatically assume that all va-riables are normally distributed. Many highly predictive biomarkers are far from normally distributed, especially in cancer research $[14,15]$.

\footnotetext{
Abbreviations

AUROCC: area under the receiver operating characteristics curve; CV: crossvalidation; DAT: data analysis team; DLDA: diagonal linear discriminant analysis; ER: estrogen receptor; MAQC-II: MicroArray Quality Control phase ॥ on predictive modeling.
}

\section{Competing interests}

The authors declare that they have no competing interests.

\section{Authors' contributions}

JC designed and implemented the proposed feature selection algorithm and performed the experiments. All authors participated in discussing and drafting the manuscripts. All authors participated to and approved the final manuscript's preparation.

\section{Acknowledgements}

We would like to thank Dr. Pankaj Agarwal, Dr. Lajos Pusztai and Dr. Lihua Yu for the invaluable suggestions and feedbacks.

\section{Declarations}

The publication costs for this article were funded by the corresponding author's institution.

This article has been published as part of BMC Systems Biology Volume 7 Supplement 4, 2013: Selected articles from the IEEE International Conference on Bioinformatics and Biomedicine 2012: Systems Biology. The full contents of the supplement are available online at http://www.biomedcentral.com/ bmcsystbiol/supplements/7/S4.

\section{Authors' details}

${ }^{1}$ Quantitative Sciences, GlaxoSmithKline, Collegeville, PA 19426, USA. ${ }^{2}$ Cancer Research, GlaxoSmithKline, Collegeville, PA 19426, USA. ${ }^{3}$ National Center for Toxicological Research, US Food and Drug Administration, Jefferson, AR 72079, USA. ${ }^{4}$ Cancer Institute, Zhejiang University, Hangzhou, 310009, China.

\section{Published: 23 October 2013}

\section{References}

1. Subramanian J, Simon R: Gene expression-based prognostic signatures in lung cancer: ready for clinical use? J Natl Cancer Inst 2010, 102:464-74.

2. Simon R: Roadmap for developing and validating therapeutically relevant genomic classifi-ers. J Clin Oncol 2005, 23:7332-41.

3. Dudoit R, Fridly J, Speed TP: Comparison of Discrimination Methods for the Classification of Tumors Using Gene Expression Data. JAMA 2002, 97457:77-87.

4. Cheng J, Greshock J, Shi L, Painter J, Lin X, Lee K, Zheng S, Wooster R, Pusztai $L$, Menius A: An adaptive feature selection method for microarray data analysis. Bioinformatics and Biomedicine (BIBM), 2012 IEEE International Conference on: 4-7 October 2012 2012, 1-6.

5. Greshock J, Cheng J, Rusnak D, Martin AM, Wooster R, Gilmer T, Lee K, Weber BL, Zaks T: Genome-wide DNA copy number predictors of lapatinib sensitivity in tumor-derived cell lines. Mol Cancer Ther 2008, 7:935-943.

6. MAQC consortium: The MicroArray Quality Control (MAQC)-Il study of common practices for the development and validation. Nat Biotechnol 2010, 28:827-838.

7. van de Vijver MJ, He YD, van't Veer LJ, Dai H, Hart AA, Voskuil DW, Schreiber GJ, Peterse JL, Roberts C, Marton MJ, Parrish M, Atsma D, Witteveen A, Glas A, Delahaye L, van der Velde T, Bartelink H, Rodenhuis S, Rutgers ET, Friend SH, Bernards R: A gene-expression signature as a predictor of survival in breast cancer. N Engl J Med 2002, 347:1999-2009.

8. Buyse M, Loi S, van't Veer L, Viale G, Delorenzi M, Glas AM, d'Assignies MS, Bergh J, Lidereau R, Ellis P, Harris A, Bogaerts J, Therasse P, Floore A, Amakrane M, Piette F, Rutgers E, Sotiriou C, Cardoso F, Piccart MJ: Validation and clinical utility of a 70-gene prognostic signature for women with node-negative breast cancer. J Natl Cancer Inst 2006, 98:1183-92.

9. van 't Veer $L J$, Dai H, van de Vijver MJ, He YD, Hart AA, Mao M, Peterse $H L$, van der Kooy K, Marton MJ, Witteveen AT, Schreiber GJ, Kerkhoven RM, Roberts C, Linsley PS, Bernards R, Friend SH: Gene expression profiling predicts clinical outcome of breast cancer. Nature 2002, 415:530-536.

10. Haybittle JL, Blamey RW, Elston CW, Johnson J, Doyle PJ, Campbell FC, Nicholson Rl, Griffiths K: A prognostic index in primary breast cancer. British journal of cancer 1982, 45(3):361-6.

11. Ravdin PM, Siminoff LA, Davis GJ, Mercer MB, Hewlett J, Gerson N, Parker HL: Computer program to assist in making decisions about adjuvant therapy for women with early breast cancer. J Clin Oncol 2001 19:980-91.

12. Esteva FJ, Sahin AA, Cristofanilli M, Coombes K, Lee SJ, Baker J, Cronin M, Walker M, Watson D, Shak S, Hortobagyi GN: Prognostic role of a multigene reverse transcriptase-PCR assay in patients with node-negative breast 
cancer not receiving adjuvant systemic therapy. Clin Cancer Res 2005, 11:3315-3319.

13. Wang $Y$, Klijn JG, Zhang Y, Sieuwerts AM, Look MP, Yang F, Talantov D, Timmermans M, Meijer-van Gelder ME, Yu J, Jatkoe T, Berns EM, Atkins D, Foekens JA: Gene-expression profiles to predict distant metastasis of lymph-node-negative primary breast cancer. Lancet 2005, 365(9460):671-9.

14. Tomlins SA, Rhodes DR, Perner S, Dhanasekaran SM, Mehra R, Sun XW, Varambally S, Cao X, Tchinda J, Kuefer R, Lee C, Montie JE, Shah RB, Pienta KJ, Rubin MA, Chinnaiyan AM: Recurrent fusion of TMPRSS2 and ETS transcription factor genes in prostate cancer. Science 2005, 310:644-8.

15. Tibshirani R, Hastie T: Outlier sums for differential gene expression analysis. Biostatistics 2007, 8:2-8.

doi:10.1186/1752-0509-7-S4-S2

Cite this article as: Cheng et al:: Good practice guidelines for biomarker discovery from array data: a case study for breast cancer prognosis. $B M C$ Systems Biology 2013 7(Suppl 4):S2.

\section{Submit your next manuscript to BioMed Central} and take full advantage of:

- Convenient online submission

- Thorough peer review

- No space constraints or color figure charges

- Immediate publication on acceptance

- Inclusion in PubMed, CAS, Scopus and Google Scholar

- Research which is freely available for redistribution

Submit your manuscript at www.biomedcentral.com/submit
C Biomed Central 